\title{
Avaliação da prevalência de idosos polifarmácia pelo Programa PET Saúde em Estratégia de Saúde da Família
}

\author{
Evaluation of the prevalence of elderly polypharmacy by the PET Health Program in the Family Health Strategy \\ Evaluación de la prevalencia de polifarmacia en ancianos por el Programa de Salud PET en Estrategia de Salud de \\ la Familia
}

\begin{abstract}
RESUMO
O aumento da expectativa de vida ao longo dos anos vem acompanhado da presença das doenças crônicas não transmissíveis e se faz necessário o uso de terapias medicamentosas associadas geralmente configurando a prática de polifarmácia. Método: Foi realizada um estudo transversal descritivo com os idosos pertencentes a uma Estratégia de Saúde da Família no interior de São Paulo onde verificou-se a informações sociodemográficas, grau de instrução, problemas de memória, uso de medicamentos, doenças mais prevalentes. Resultados: A maioria dos idosos possuíam comorbidades, além disso pequena parcela $(0,8 \%)$ não usavam medicamentos continuamente, observou-se uma predileção ao perfil de polifarmácia entre os 60 e 74 anos. Conclusão: A abordagem interprofissional pode ser uma estratégia efetiva visando a promoção do uso racional de medicamentos, onde o trabalho colaborativo da equipe de saúde pode fornecer ao paciente orientações específicas e adequadas à sua terapia medicamentosa, para a efetividade do tratamento e sua segurança.
\end{abstract}

DESCRITORES: Educação Interprofissional; Saúde Pública; Saúde do Idoso.

\begin{abstract}
Introduction: The increase in life expectancy over the years is accompanied by the presence of chronic non-communicable diseases and the use of associated medicine therapy is necessary, usually it is configuring the polyfarmacy practice. Methods: A descreptive cross-sectional study was performed with de elderly belonging to a Family Heatlh Strategy in the São Paulo interior, where sociodemographic information, education level, memory problems, medication use, and the most prevalent diseases were found. Results: Most of the elderly had comorbidities, moreover, in a small portion ( $0.8 \%$ didn't use medication continuously, there was a predilection for the polypharmacy profile between 60 and 74 years old. Conclusion: The interprofessional approach can be an effective strategy aiming the rational use of medicines promotion, where the collaborative work of the health team can supply to patient specific and adequate exposure for their drug therapy, the treatment effectiveness and yours safety. DESCRIPTORS: Interprofessional Education; Public Health; Elderly Health.
\end{abstract}

\section{RESUMEN}

El aumento de la esperanza de vida a lo largo de los años ha ido acompañado de la presencia de enfermedades crónicas no transmisibles y es necesario el uso de terapias farmacológicas asociadas, configurando generalmente la práctica de la polifarmacia. Método: Se realizó un estudio descriptivo transversal con ancianos pertenecientes a una Estrategia de Salud de la Familia en el interior de São Paulo, donde se verificó la información sociodemográfica, nivel educativo, problemas de memoria, uso de medicamentos, enfermedades más prevalentes. Resultados: La mayoría de los ancianos presentaba comorbilidades, además, una pequeña porción $(0.8 \%$ ) no usaba medicamentos de manera continua, existía predilección por el perfil de polifarmacia entre los 60 y 74 años. Conclusión: El abordaje interprofesional puede ser una estrategia eficaz orientada a promover el uso racional de los medicamentos, donde el trabajo colaborativo del equipo de salud puede brindar al paciente una orientación específica y adecuada para su farmacoterapia, para la efectividad del tratamiento y su seguridad.

DESCRIPTORES: Educación Interprofesional; Salud pública; Salud de los ancianos.

RECEBIDO EM: 30/08/2020 APROVADO EM: 11/09/2020 


\section{artigo}

Fagundes, A.C.G.; Negrini, L.D.O.; Rodrigues, R.F.O.; Oliveira, C.B.S.J.; Alves, C.B.; Oliveira, J.L.; Marques, L.S.;

\section{Ana Carolina da Graça Fagundes}

Possui graduação em Odontologia pela Faculdade de Odontologia de Araçatuba-UNESP(2008). É Mestre(2011) e Doutora(2015) em Odontologia Preventiva e Social pela Faculdade de Odontologia de Araçatuba-UNESP; Especialista em Atenção Básica e Saúde da Família pela Universidade Federal de São Paulo-UNASUS/UNIFESP(2015). Professora convidada no curso de Odontologia da Universidade São Francisco (USF)-campus de Bragança Paulista(2017-atual). Coordenadora do projeto do Centro de especialidade Odontológicas USF. Tutora Coordenadora de GAT do Projeto PETSaúde Interprofissionalidade 2019/2021 (desde 2019).

ORCID: 0000-0002-0110-0615

\section{Lisamara Dias de Oliveira Negrini}

Possui graduação em Enfermagem pela Universidade São Francisco (2008); Especialização em Gestão em Saúde pela Universidade São Francisco (2010); Especialização em Auditoria em Serviços de Saúde pela Universidade Nove de Julho (2016); e Especialização em Preceptoria no SUS pelo Instituto de Ensino e Pesquisa Hospital Sírio Libanês (2017); Cursando Mestrado Profissional no Programa de Pós Graduação em Ciências da Saúde do Centro de Desenvolvimento do Ensino Superior em Saúde (CEDESS) da Universidade Federal de São Paulo (UNIFESP). Enfermeira na Secretaria Municipal de Saúde de Bragança Paulista (desde 2009): Gerente Local e Assistencial na Atenção Primária em Saúde (até 2011); Coordenadora do Componente Municipal de Auditoria do SUS (desde 2012); Coordenadora do Núcleo de Educação Permanente em Saúde e Articuladora do COAPES Regional (desde 2016); Coordenadora do Projeto PETSaúde Interprofissionalidade 2019/2021 (desde 2019). Docente no Ensino Técnico (até 2011). Docente Convidada na Universidade São Francisco (desde 2015): Compõem o Núcleo de Saúde Pública (desde 2015); Articuladora pedagógica para práticas em saúde e Integração Ensino/Serviço/Comunidade. ORCID: 0000-0001-5165-7967

\section{Rosa Fátima de Oliveira Rodrigues}

Graduada em Farmácia pela Universidade de São Paulo - USP - Ribeirão Preto, Doutorado em Fármacos e Medicamentos Área de Insumos Farmacêuticos pela Universidade de São Paulo-USP-São Paulo, Especialista em Homeopatia pelo Instituto François Lamasson-Ribeirão Preto.

ORCID: 0000-0003-4357-7507

\section{Carolina Bernardi Stefani}

Farmacêutica e Bioquímica, especialista em Saúde Pública, precpetora do Programa PET Saúde.

ORCID: 0000-0002-6783-1437

\section{Jane de Oliveira}

Graduada em Enfermagem pela Universidade São Francisco, pós graduada em Auditoria em Enfermagem pela Universidade São Camilo. ORCID: 0000-0002-1017-8748

\section{Clara Brito Alves}

Graduada em Enfermagem pela Universidade São Francisco, aluna bolsista do Programa PET Saúde no período de abril de 2019 à abril de 2020.

ORCID: 0000-0002-2725-9165

\section{Juliana Oliveira Leite}

Aluna do $6^{\circ}$ semestre do Curso de Biomedicina na Universidade São Francisco - Campus Bragança Paulista, aluna bolsita no Projeto PET Saúde desde abril de 2019.

ORCID: 0000-0002-4944-0272

\section{Leonardo de Souza Marques}

Aluno do $8^{\circ}$ semestre do Curso de Odontologia da Universidade São Franscisco - Campus Bragança Paulista, aluno bolsista no Programa PET Saúde, desde abril de 2019.

ORCID: 0000-0002-3379-2388

\section{INTRODUÇÃO}

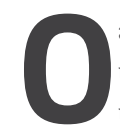
aumento da expectativa de vida é uma das grandes conquistas das últimas décadas, fato relacionado a melhora significativa das condições de saúde da população em todo o mundo. No Brasil a expectativa mostra-se crescente, atingindo 76,3 anos em 2018, 30,8 anos a mais do que o observado em 1940. Para indivíduos do sexo feminino, estima-se maior longevidade: 79,9 anos, e 72,8 anos para o sexo masculino. Essa diferença é mais acentuada conforme a faixa etária e esses dados variam ainda conforme as regiões país ${ }^{1}$.

No ano de 2017, a população das Américas com mais de 60 anos de idade era de 
14,6\%, e na América Latina essa proporção deverá se aproximar de $25 \%$ até 2050.

$\mathrm{Na}$ Europa o envelhecimento da população levou aproximadamente 65 anos para ocorrer, no Canadá e Estados Unidos, cerca de 75 anos. Nas Américas essas mudanças ocorrerão em apenas 35 anos, ou seja, metade do tempo observado para adaptação às mudanças se comparadas a outras regiōes do mundo, uma vez que para muitos o envelhecimento vem acompanhado por doenças crônicas e deficiências que, em muitos casos, afetam a capacidade das pessoas de serem autossuficientes ${ }^{2}$.

No Brasil, $70 \%$ dos idosos possuem pelo menos uma patologia crônica, necessitando de tratamento farmacológico regular para controle dessas patologias. De acordo com Silva $(2012)^{3}$ e Pereira $(2017)^{4}$ as doenças crônicas relacionadas com a idade, como dislipidemia, hipertensão e diabetes, requerem geralmente o uso de múltiplas drogas, de modo que 56\% dos idosos possuem receitas com mais de quatro medicamentos de uso contínuo. No estudo de Rodrigues e Oliveira (2016) estima-se que $40 \%$ dos idosos usam 5 ou mais, e $12 \%$ usam 10 ou mais medicamentos diferentes 5 .

Embora não haja consenso na literatura quanto à quantidade de medicamentos necessária à configuração da polifarmácia, em geral, aceita-se o uso de 5 medicamentos ou mais ao mesmo tempo. Bermudez (2010) por sua vez relaciona ainda, o tempo de consumo dos medicamentos por um período de 60 a 90 dias, porém ressalta que outros autores consideram períodos superiores a $15 \mathrm{dias}^{6}$.

Ramos et al (2016) ${ }^{7}$, em estudo sobre a prevalência de polifarmácia identificaram que $91 \%$ dos idosos fazem uso de algum fármaco, e que $27 \%$ utilizavam cinco ou mais. Já o estudo de Silva et al $(2018)^{8}$ observou que além das comorbidades, estão implicados na gênese da polifarmácia o número de médicos consultados, a ausência de perguntas sobre os medicamentos em uso durante a consulta médica e a automedicação.

Neste sentido, a polifarmácia representa excesso de custo para o Sistema Único de Saúde (SUS) e uma maior carga finan-
Embora não

haja consenso na

literatura quanto

à quantidade de

medicamentos

necessária à

configuração da

polifarmácia, em

geral, aceita-se o uso

de 5 medicamentos

ou mais ao mesmo

tempo. Bermudez

(2010) por sua vez

relaciona ainda, $o$

tempo de consumo

dos medicamentos

por um período de

60 a 90 dias, porém

ressalta que outros

autores consideram

períodos superiores a

15 dias. ceira aos idosos. Pode contribuir para o uso de medicamentos inadequados e não essenciais, criando uma barreira para a adesão ao tratamento, na medida em que tornam mais complexos os esquemas terapêuticos, além de favorecer interações medicamentosas e reações adversas. Uma comunicação eficiente entre esses profissionais e a coordenação entre vários prescritores é crucial para o sucesso da terapêutica, além disso, programas educativos para melhorar o hábito da prescrição racional e a educação do paciente no momento da alta e do seguimento são importantes estratégias 5 .

Em 2018, o projeto "PRÁTICA COLABORATIVA E ATENÇÃO INTEGRAL NOS CICLOS DE VIDA" elaborado pela Universidade São Francisco em parceria com a Secretaria Municipal de Saúde de Bragança Paulista foi aprovado no Programa de Educação pelo Trabalho para a Saúde (PET-Saúde) Interprofissionalidade que é uma estratégia dos Ministério da Saúde e da Educação para a transformação da formação profissional em saúde, com maior integração entre ensino, serviço e comunidade, com vistas a incorporação da Educação Interprofissional (EIP) ao currículo dos cursos da área da saúde e a Prática Colaborativa (PC) aos serviços de saúde ${ }^{9,10}$.

Entendida como ocasião em que membros de duas ou mais profissóes aprendem em conjunto, de forma interativa, a EIP tem o propósito de melhorar a qualidade da atenção dos indivíduos, famílias e comunidade $^{11}$. Nesta proposta, sugere-se que alunos aprendam de forma interativa sobre papéis, conhecimentos e competências dos demais profissionais desenvolvendo subsídios para fortalecer o trabalho em equipe, tendo em vista a transformação das práticas de saúde, desafiando o contexto usual de formação para encontrar respostas novas a novos problemas ${ }^{12}$.

Com início das atividades em abril de 2019, o Projeto que foi organizado em 04 (quatro) Grupos de Aprendizagem Tutorais (GAT) e propôs atuar na Atenção Primária a Saúde (APS), nos diferentes ciclos de vida - criança e adolescente, mulher e gestante, adulto e idosos. Neste contex- 
to, o GAT2, composto por 2 docentes, 2 preceptores (profissionais da saúde) e 6 discentes de diferentes áreas profissionais vêm atuando com os idosos pertencentes à Estratégia de Saúde da Família(ESF) Vila Motta, com vistas ao desenvolvimento de práticas cotidianas permitam maior integração entre ensino, serviço e comunidade para assim superar a fragmentação do trabalho em saúde e da formação de profissionais de saúde.

Este artigo objetiva apresentar o diagnóstico realizado pelo GAT2, no âmbito do PET Saúde Interprofissionalidade junto a população idosa pertencente a ESF Vila Mota em Bragança Paulista sobre o acometimento de doenças e a frequência do uso de medicamentos, classificando-os como polifarmácia (quando do uso de 5 ou mais medicamentos) observando também a autonomia dos idosos e problemas relacionados à saúde geral e mental.

\section{MÉTODO}

Trata-se de um estudo transversal descritivo, para traçar um perfil epidemiológico da população a ser estudada. A amostra foi determinada por conveniência e o projeto foi submetido e aprovado pelo comitê de ética (CAAE 13440719.800005514). A coleta de dados foi realizada por meio de um questionário online (Google forms), baseado na cartilha do idoso, o qual abordou questões relacionadas ao perfil socioeconômico e cultural, padrão de dependência e de saúde e doença dos idosos no último ano. A coleta de dados se deu entre os meses de junho e julho de 2019, sendo utilizado como critério de inclusão os idosos que pertencem a unidade de saúde e que quando abordados em sala de espera, durante a dispensação de medicamentos e em visitas domiciliares consentiram em participar por meio do TCLE, sendo respeitado o sigilo de suas identidades.

\section{RESULTADOS}

A ESF da Vila Mota apresentou os 817 idosos cadastrados e desse total 127 idosos consentiram em participar da pes-

\section{Dentre os 817}

idosos cadastrados

na ESF estudada,

participaram da

pesquisa 127 idosos,

o que representa

$15,5 \%$ do total.

$\mathbf{6 8 , 5 \%}$ pertencem

ao sexo feminino

e $31,5 \%$ ao sexo

masculino.

quisa(15,5\%). Do total de participantes a maioria era do sexo feminino $(68,5 \%)$. No que se refere às faixas etárias, $63,8 \%$ se encontravam com 60 a 74 anos, entre 75 a 84 anos, cerca de $30,7 \%$ e com 85 anos ou mais representaram 5,5\%. Em relação as informações socioeconômicas $85 \%$ afirmam receber aposentadoria ou algum benefício e $91,3 \%$ se declaram alfabetizados. $37 \%$ referem viver sozinhos e não possuir companheiro(a) e $20,5 \%$ referem depender de cuidador. Cerca de $26,8 \%$, relatam ter falta de memória e as doenças prevalentes foram Diabetes Mellitus (44,09\%), Hipertensão Arterial (66,14\%) e Acidente Vascular Cerebral (AVC) 6,29\%. No que tange a saúde mental, 21,25\% apresentam depressão e 2,36\% quadros demenciais. Sobre o uso contínuo de medicamentos apenas $0,8 \%$ negam a utilização, 19,7\% afirmam usar até duas drogas diferentes, $37 \%$ até cinco e $42,5 \%$ cinco ou mais. Cerca de $17,3 \%$ referem dificuldade em tomar corretamente os medicamentos.

\section{DISCUSSÃo}

O elevado percentual de idosos na faixa de idade de 60 a 74 anos pode estar relacionado a uma maior independência, procura por cuidados de saúde e consequente maior utilização/prescrição de medicamentos, observando-se que polifarmácia se apresenta em grande parte do grupo que refere o uso de cinco ou mais medicamentos contínuos.

Dentre os 817 idosos cadastrados na ESF estudada, participaram da pesquisa 127 idosos, o que representa $15,5 \%$ do total. $68,5 \%$ pertencem ao sexo feminino e $31,5 \%$ ao sexo masculino. No que se refere às faixas etárias, $63,8 \%$ se encontravam com 60 a 74 anos, entre 75 a 84 anos, cerca de $30,7 \%$ e com 85 anos ou mais representaram 5,5\%. 85\% afirmam receber aposentadoria ou algum benefício e $91,3 \%$ se declaram alfabetizados. $37 \%$ referem viver sozinhos e não possuir companheiro(a) e $20,5 \%$ referem depender de cuidador.

Sobre o uso contínuo de medicamentos apenas $0,8 \%$ negam a utilização, $19,7 \%$ afirmam usar até duas drogas diferentes, $37 \%$ até cinco e $42,5 \%$ cinco ou mais. Cerca de $17,3 \%$ referem dificuldade em tomar corretamente os medicamentos. Quando considerado o sexo, observou-se a prevalência de polifarmácia em mulheres.

Cerca de $26,8 \%$, relatam ter falta de memória e as doenças prevalentes foram Diabetes Mellitus (44,09\%), Hipertensão Arterial (66,14\%) e Acidente Vascular Cerebral (AVC) 6,29\%. No que tange a saúde mental, 21,25\% apresentam depressão e 2,36\% quadros demenciais.

Para Nascimento et al $(2017)^{13}$ a polifarmácia tem ocorrido na prática clínica de forma comum e crescente, principalmente em pessoas acima de 65 anos. Este crescimento relaciona-se a vários fatores, como o aumento da expectativa de vida e o consequente aumento da multimorbida- 
de, à maior disponibilidade de fármacos e diretrizes que recomendam o uso de associações medicamentosas para o manejo de várias condições de saúde, como a Hipertensão Arterial e o Diabetes Mellitus.

A participação de equipe interdisciplinar na assistência aos pacientes bem como o treinamento da equipe quanto à indicação e prescrição de medicamentos e a disponibilidade de medicamentos adequados para os idosos podem elevar a qualidade do serviço fornecido e reduzir a ocorrência de fatores adversos, obtendo-se assim uma terapia eficaz e melhor qualidade de vida aos idosos ${ }^{14}$.

Esta pesquisa proporcionou aos atores envolvidos estudantes, preceptores, tutores e coordenação do Projeto PET-Saúde Interprofissionalidade, trabalhar as competências colaborativas na prática por meio da vivência dos desafios existentes nos serviços de saúde, olhar interprofissional e as habilidades necessárias para o desenvolvimento de ações de saúde apropriadas a demanda da população estudada ${ }^{15}$.

\section{CONCLUSÃO}

A abordagem interprofissional pode ser uma estratégia efetiva visando a promoção do uso racional de medicamentos, onde o trabalho colaborativo da equipe de saúde pode fornecer ao paciente orientações específicas e adequadas à sua terapia medicamentosa, para a efetividade do tratamento e sua segurança. $O$ processo de envelhecimento gera desafios para toda a sociedade, e as equipes de saúde da família tem papel fundamental pela sua relação de proximidade com os pacientes, famílias e comunidade, assim como com a gestão pública de saúde.

A associação entre uso de medicamentos inadequados e variáveis como nível socioeconômico, visita a serviços de saúde e uso de medicamentos prescritos comporta várias explanações. Pode refletir o desconhecimento do profissional médico em relação à prescrição de medicamentos não recomendados para idosos, como também a prescrição de medicamentos que estão mais disponíveis nos serviços públicos, muitos deles considerados inadequados.

Para que as propostas de intervenção tenham o impacto necessário entre a população idosa é importante conhecer as especificidades desta população de acordo com o território onde vivem e assim poder traçar estratégias de melhorias que possam ser usadas pela equipe.

\section{REFERÊNCIAS}

1. IBGE - Instituto Brasileiro de Geografia e Estatística [Internet]. Brasil; c2018 [Acesso em 24 ago 2020]. Disponível em: https:/censo2020. ibge.gov.br/2012-agencianoticias/noticias/26103expectativa-de-vida-dos-brasileiros-aumenta-para-76-3-anos-em-2018.html

2. ONU - Organização das Nações Unidas [internet]. Nova lorque; c2019 [Acesso 24 ago 2020]. Disponivel em: https://news.un.org/pt/ story/2019/06/1676601.

3. Silva R, Schmidt OF, Silva S. Polifarmacia em geriatria. Revista da AMRIGS. 2012; 56 (2): 164-174,

4. Pereira KG, Peres MA, lop D, Boing AC, Boing AF, Aziz M, et al. Polifarmácia em idosos: um estudo de base populacional. Rev. Bras. Epidemiologia [internet]. 2017jun [Acesso em 24 ago 2020]; 20(2): 335-344. Disponível em: https://doi.org/10.1590/19805497201700020013.

5. Rodrigues MCS, Oliveira, C. Interações medicamentosas e reações adversas a medicamentos em polifarmácia em idosos: uma revisão integrativa. Rev. Latino-Am. Enfermagem. [Internet]. 2006abr [Acesso em 24 ago 2020]; 24:1-17 Disponível em: https://www.scielo.br/pdf/rlae/v24/pt_0104-1169-rlae-24-02800.pdf

6. Bermudes M. Renda, escolaridade, ir acompanhado na consulta, morar sozinho, o que é mais importante para que o idoso siga as prescrições médicas? Revista Científica. 2010; 5: 94-6.

7. Ramos LR, Tavares NUL, Bertoldi AD, Farias MR, Oliveira MA, Luiza VL, et al. Polifarmácia e polimorbidade em idosos no Brasil: um desafio em saúde pública. Rev. Saúde Pública. 2016; 50 (supl 2): 9s.

8. Silva MRR, Diniz LM, Santos JRB, Reis EA, Mata RA, Araújo VE, et al. Uso de medicamentos e fatores associados à polifarmácia em indivíduos com diabetes mellitus em Minas Gerais, Brasil. Ciência \& Saúde Coletiva. 2018, 23: 2565-2574
9. Ministério da Saúde (BR). Secretaria de Gestão do Trabalho e da Educação na Saúde.Departamento de Gestão da Educação na Saúde. Relatório final da oficina de alinhamento conceitual sobre educação e trabalho interprofissional em saúde. Brasília (DF); 2017.

10. Ministério da Saúde [internet]. Brasil; c2019 [Acesso em 21 abr 2019] Ministério da Saúde inicia as atividades do PET-Saúde/ Interprofissionalidade. Disponivel em: http://portalms.saude.gov. br/noticias/sgtes/44846-ministerio-da-saude-inicia-as-atividades-do-pet-saude-interprofissionalidade.

11. Reevs S, Fletcher S, Barr H, Birch I, Boet S, Davies N, et al. A BEME systematic review of the effects of interprofessional education: BEME. 2006; 38(7): 656-6

12. Peduzzi, M, Norman IJ, Germani ACCG, Silva, JAM, Souza, CG. Interprofessional education: training for healthcare professionals for teamwork focusing on users. Revista da Escola de Enfermagem da USP [Internet] 2013 aug [Acess 2020 aug 24]; 47(4):973-9. Disponível em: https://doi.org/10.1590/S0080623420130000400029

13. Nascimento RA, Alvares J, Junior AAG, Gomes, Cl, Silveira, MR, Costa EA, et al. Polypharmacy: a challenge for the primary health care of the Brazilian Unified Health System. Revista de Saúde Pública. $2017 ;$ 51(2):1-22.

14. Silva, EA, Macedo, LC. Polifarmácia em idosos. Revista Saúde e Pesquisa. 2013, 6(3): 477-86.

15. Almeida, RGS, Teston, EF, Medeiros AA. A interface entre o PET-Saúde/Interprofissionalidade e a Política Nacional de Educação Permanente em Saúde. Saúde debate [Internet]. 2019ago [Acesso 30 ago 2020] 43(spe1): 97-105. Disponível em: https://doi. org/10.1590/0103-11042019s108 